\title{
A framework for improving diversity work in physics
}

\author{
Geraldine L. Cochran \\ Department of Physics and Astronomy I Office of STEM Education, \\ Rutgers University, 136 Frelinghuysen Rd, Piscataway, NJ, 08854
}

\author{
Mildred Boveda \\ Mary Lou Fulton Teachers College, Arizona State University, PO Box 871811, Tempe, AZ, 85287
}

In this paper we draw on Black Feminist Theory to motivate examining diversity work in physics with an emphasis on physics education research. In our framework, we consider three major approaches to doing diversity work acknowledging and naming diversity, affirming diversity, and removing barriers to increase diversity. We examine these approaches with goals of attending to the affordances of existing diversity work and identifying the constraints for those currently seeking to diversify the field. We use intersectionality to critique diversity efforts in physics and conclude with suggestions on elevating and complicating diversity work in physics and physics education research. 


\section{INTRODUCTION}

Established in the 1970s, physics education research (PER) is a relatively new field [1]. Over the past two decades, there has been increasing attention to diversity in PER, and the broader field of physics. As used in this paper, diversity refers to the representation of who accesses and engages with physics based on sociocultural, sociohistorical, and sociopolitical factors. There are numerous sociocultural categories that societies use to describe people that have been contextually and historically constructed as other. For each category (e.g. nationality, race, ethnicity, gender, disability, sexuality), there are markers that are considered the "normative" or "dominant" identities. Identities outside of these dominant markers represent markers of difference. Diversity thus constitutes members of a community who have one or multiple markers of difference. These markers may influence an individual's perception of self [2], expected performance in educational settings [3], and their interactions with others [4].

Physicists are "doing diversity work" in a variety of ways. In this paper, we articulate the importance of interrogating how physicists are doing diversity work. We turn to Black Feminist Theory [5] to argue for why rethinking diversity work is important and use intersectinoality theory [6] to offer suggestions for how physicists might move forward in ways that are inclusive of all differences. The intended audience for this paper is the PER community; thus, we draw heavily from examples in PER and at the organization level, include examples from the American Association of Physics Teachers (AAPT), the American Physical Society (APS), and the American Institute of Physics (AIP).

\section{DIVERSITY WORK IN PHYSICS}

We consider three major approaches to doing diversity in this paper: naming and acknowledging diversity, affirming diversity, and removing barriers to increase diversity in physics. We posit that each of these ways of doing diversity work is unique and results in a distinctive impact on the field. In talking about the multi-faceted ways that attending to diversity matter, Crenshaw - "a leading authority in Black feminist legal theory ... and racism" [7] explains that "the better we understand how identities and power work together from one context to another, the less likely our movements for change are to fracture [8]." The purpose of examining these three approaches, therefore, is to explicitly attend to the affordances of existing diversity work and identify the constraints for those currently seeking to diversify the field.

\section{A. Naming and acknowledging diversity}

One of the ways that the PER community has engaged in diversity work is by naming and acknowledging - through public discourse - the differences in experiences of those underrepresented, minoritized, and marginalized in physics and PER. Whether it is naming the need for diversity work in K-12 schooling, undergraduate classrooms, and graduate schools or acknowledging the lack of diversity within professional, academic, and scholarly physics communities, there is evidence that physicists are paying attention to diversity.

For example, a review of the of 2018 and 2019 Physics Education Research Conference Proceedings revealed that a total of 35 of the 225 (15.6\%) explicitly mentioned the gender, race, ethnicity, sexuality, nationality, socio-economic status, and/or disability of participants in the abstract. Additionally, this work is included in themed issues and focused collections related to diversity in physics. Examples include the focused collection on gender in physics presented by Brewe and Sawtelle [9], and the themed collections on race and physics teaching [10] and sex, gender, and physics [11] presented by Cochran and White. Another example would be the resource letter on Gender and Physics published by the American Journal of Physics [12]. Physicists also engage in public discourse on diversity through non-peer reviewed online articles, blogposts, and discussions on social media. Examples of these include Prescod-Weinstein's article on the inclusion of Black people in physics [13], Rodriguez's article on her decision to leave her PhD program in physics [14], Cochran's article on diversity, equity, and inclusion terminology [15], and Perry's article on her unrequited love for physics [16].

Physics professional societies have participated in naming and acknowledging by means of newsletters and special reports. For example, APS has published The APS Gazette [17], a newsletter of the APS Committee on the Status of Women and Physics since 1981 and, since 2011, a newsletter of the APS Committee on the Status of Women in Physics and the Committee on Minorities. In 2016, APS published a report on LGBT climate in physics [18] and in 2020 AIP published a report on African Americans pursuing bachelor's degrees in physics and astronomy [19]. The AIP's Statistical Research Center regularly publishes reports on physics related to gender and race. Another example of naming and acknowledging is AAPT's website of diversity resources [23] including a video series on women in physics [24]. We offer as one final example, the STEM inclusion studies of the AAPT [25] and the APS [26] which examined the experiences of its members with attention to members identifying as women, ethnic/racial minorities, persons with disabilities, and lesbian, gay, bisexual, transgender, and queer.

Some have argued that naming and acknowledging diversity in education, though an important first step, is not enough $[15,27]$. In many respects, we argue, that holds true within the physics community. While naming and acknowledging alone do not result in sufficient progress, defining and operationalizing diversity by first naming it helps to focus on actionable steps. Naming starts conversations by signaling who longs for greater diversity in the field and by bringing awareness to those who, due to their privileged positionalities, are oblivious to the lack of diversity. These conversations have the potential to be the catalyst needed for change. 
Examining the naming of diversity work in physics also reveals what aspects of the conversation are missing. As one example, the public discourse on disability and physics was absent for some time, but is beginning to increase [28-32]. As another example, the field is not tracking sexuality very well. There are no statistical reports on LGBTQ physicists and gathering such would be difficult as LGBTQ physicists "face uneven protections with regard to employment" and "in physics environments, social norms establish expectations of closeted behavior" [18]. There are also gaps in the literature on the experiences of physicists from lower socioeconomic backgrounds or the physics trajectories for those contending with financial disadvantages. Yet, there is evidence socioeconomic status and lack of financial support impact access and participation in physics [19, 33]. As a final example, there is a failure to appropriately name and acknowledge intra-group differences and instead a preference to use panethnic terms such as Asian, or Latino/a/x. When it comes to Asian immigrants and Asian Americans [20], for example, the use of panethnic terms does not allow for acknowledging that Asians "are a complex and changing population" that is comprised of "multicultural, multilingual people who hold different worldviews and divergent modes of interpretation [20]." In 1990, "the census identified fifty-seven groups" that were included in the Asian Pacific Islander umbrella category [21]. Moreover, this lack of acknowledgement, naming, or counting of these groups in physics has resulted in the entire panethnic group of Asian being labeled as overrepresented. The authors are unaware of any tracking of the participation of these groups in physics; however, in higher education we found that college enrollment was much higher (over 50\%) for Chinese Americans and Japanese Americans, and much lower (below 30\%) for Native Hawaiians and Laotian Americans [21]. Of course, the use of the panethnic terms Asian and Latinx has historical connections to the socially constructed categories of race and racism in the United States [22].

Examining the acknowledging and naming of diversity work provides some direction on what is needed to move the field forward. Statistical reports and numbering - which we classify as naming - is a forward step, but are insufficient to capturing the full range of differences in this field or the vulnerability that comes with differences. Indeed, some people choose to hide their difference because there is evidence of attack, marginalization, isolation, and increased vulnerability. Consequently, physicists must also consider ways to engage in diversity work that is beyond naming and acknowledging. In particular, physicists should also affirm diversity.

\section{B. Affirming diversity}

There are numerous ways to affirm diversity [34]. Affirming diversity might involve individual action, interpersonal interactions, collective action, or institutional/organizational action. Affirming diversity often means affirming some sociopolitical aspect of one's own identity or supporting, com- plementing, or replicating actions that affirm another person's identity. This can be evident in the language used to refer to minoritized communities. When someone from the dominant group uses the language of individuals from the minoritized group, the person from the dominant group is affirming diversity. For example, "Chicano is a self-affirming and political term reflecting the culture and realities of urban, economically oppressed Mexican Americans in U.S. society, and it grew out of the 1960s Brown Power Movement" [34]. In writing, a Chicanx individual may choose to use the less political term Mexican American, the self-affirming term Chicano, or the more affirming term Chicanx, which affirms both ethnic/cultural identity and various gender identities. When someone who is not Chicanx uses the term, they are affirming diversity. Affirmations should be authentic. As such, not all individuals will affirm the same things. Returning to the use of Chicano/a/x as an example; not all Mexican American activists choose to use Chicano/a/x or some may use it in some instances and not others $[34,35]$. So the idea is not for people of the dominant group to police the language used by individuals from minoritized groups, but to affirm diversity by using the language of minoritized individuals. Caution is also warranted when it comes to terminology, as people that share identities are not a monolithic. Even scholars marginalized in physics have differing viewpoints regarding terminology. At the individual level, affirming - especially self-affirmation is based on a desire to elevate or show pride in/for a particular identity. There are also ways to affirm diversity through institutional, organizational, and collective actions.

Some institutions of higher education have engaged in affirming diversity by supporting the work of affinity groups and professional societies led by individuals from minoritized and marginalized groups. For example, an institution might affirm racial/ethnic diversity by supporting organizations such the National Society of Black Physicists, the National Society of Hispanic Physicists, the Society for Advancing Chicanos/Hispanics and Native Americans in Science, or the American Indian Science and Engineering Society. Support may come in the form of making donations, attending meetings/conferences as exhibitors, sending students to participate in meetings/conferences, or posting job on these organizations' websites. All of these actions affirm the need for and the work of these organizations. Another way that institutions of higher education can - and have - affirmed diversity is through statements included in their job postings or application instructions encouraging individuals from marginalized and minoritized groups to apply. This form of affirmation acknowledges the rights of these individuals to access these spaces, resources, or opportunities. A third example is requiring diversity statements as a part of faculty candidate applications or student admissions applications. This provides an opportunity for the applicant to share how they engage in diversity work and it affirms the call from marginalized people that everyone be held accountable for doing diversity work and not just those from marginalized and minoritized groups. Institutions and individuals also affirm diversity when they 
become familiar with and follow the guidelines prepared by people from minoritized and marginalized groups. One example in physics is the LGBT+ Inclusivity in physics and astronomy: A best practices guide [36] and an example in PER is the document "Emerging reflections from the People of Color (POC) at PERC Discussion Space [37]. As with terminology caution is warranted; it is best to keep in mind that "best practices" are best within specific context and time and even then "best" may be subjective.

At the organizational level, physics professional societies have affirmed diversity through creation/support of committees and groups such as AAPT's Committee on Diversity and Committee on Women in Physics, APS's Committee on the Status of Women in Physics and Committee on Minorities, APS Women in Physics Groups, the recently established APS Forum on Diversity and Inclusion, and AIP's National Task Force to Elevate African American Representation in Undergraduate Physics Astronomy (TEAM-UP). This list is not meant to be exhaustive, but rather it provides some examples of committees and teams in physics that affirm diversity. Another way that professional societies have affirmed diversity is through board statements [38]. Statements affirming diversity occur at the organizational level and some occur at the committee level such as the AAPT's Committee on Laboratories' value statement on diversity and community [39]. These committees and statements affirm the importance of and acknowledge the experiences - and associated inequities - related to the sociocultural factors they address and demonstrate a commitment to addressing these issues. Some societies also host a list of women speakers and/or a list of speakers from minoritized ethnic/racial groups [40].

Collective actions of affirmation are often spontaneous and arise from a pressing need. For example, nearly 2500 physicists signed a response to the Justices of the Supreme Court of the United States [41] when Chief Justice Scalia posited that most Black students should probably go to a "lesser" school where they will succeed and Chief Justice Roberts asked "What unique perspective does a minority student bring to a physics class" [42]? Responding to commentary that calls into question individuals rights to be in certain spaces is an example of affirmation. Another example, would be physicists participation in and organization of \#Strike4BlackLives, \#ShutDownStem, and \#ShutDownAcademia strikes on June $10,2020[43,44]$ in response to racialized violence and antiBlack racism. This movement affirmed the unrecognized rights of Black people to live free of violence and racism and to exist in spaces in STEM and academia. There have also been instances where physicists have organized with others in support of affirming difference. For example, PrescodWeinstein organized a letter to Chennel "Jazzy" Rowe and other bullied Black women affirming their right to exist without anti-Black hatred [45]. That letter was signed by many Black women physicists.

Affirmation is an important aspect of diversity work because it can directly impact the numbers of people from marginalized groups participating in physics and choosing to remain in physics spaces. However, it also reveals a tension between trying to push members of the community and potentially losing members of the community. In thinking about affirming diversity, some worry about offending colleagues and retribution. As an example, some individuals engaged in diversity work in physics are comfortable saying that Black Lives Matter and others are not. The way that diversity has been affirmed reveals the tension between pushing the conversation and being radical versus going slowly and bringing others along. Some physicists advocate for only addressing issues for which there is a consensus - the "safe topics." Deciding what should be in the physics - and particularly the PER - space is a question of affirmation. For example, some physicists do not feel they should be activists and involved in political discussion. Others, acknowledge that addressing diversity in STEM requires addressing all of the issues that potential members of the community face [46].

\section{Removing barriers}

Within PER, there are efforts to both study and actively remove barriers to increasing diversity in physics. When thinking of sociopolitical and sociohistorical aspects of diversity work, it is instructive to remember that politics have been defined as the social processes that determine who gets what, when, and how [47]. Given the historical and systematic exclusion of women, People of Color, and many other minoritized groups from accessing higher education, and given the demonstrably exploitative dynamics that scientists have had with Communities of Color, it is important to locate any remnants of policies and gatekeeping practices that privilege the presence, epistemology, and dominance of white men in the field [50]. It is also critical to reflect on sociohistorical factors that prevent people from bringing their full selves to physics.

Previous research indicates that the Graduate Record Exam is a barrier to participation in graduate education [33, 51-53]. The statement of AAPT recommending that the GRE be eliminated from the admissions process is an effort to remove the barrier [54]. The APS Bridge program is designed to increase the number of physics PhDs "awarded to African American, Hispanic American, and Native American students" [55] and aspects of this program, including the common application, are aimed at removing barriers to participation [33].

Only a couple examples are listed in this paper, but there are more examples of physicists doing the work of removing barriers in physics. However, barriers are often quite difficult to remove. Despite research indicating that the use of the GRE in admissions is a hindrance to diversity in graduate programs, professional society statements encouraging that it not be used in the admissions process, and guidelines from the Educational Testing Service (ETS) [56] - the creators of the GRE tests - on how tests results should and should not be used, research indicates that many departments continue to use the GRE tests in the admissions process [57] and contrary to the guidelines given by the ETS. 


\section{ELEVATING AND COMPLICATING DIVERSITY WORK}

This framework of examining diversity work in physics allows us to assess progress in the field. For example, it highlights that there are some markers of difference that are being acknowledged and named, and others that are not. There are tensions within the PER community and the larger physics community that dictate how these communities affirm diversity. Finally, barriers to participation in the field are difficult to remove. There is a need to elevate and complicate the diversity discussion. The physics community must track and evaluate how it engages in diversity efforts and its progress. The community must do diversity well and consistently.

\section{A. Intersectionality}

One of the clear challenges we recognized in the diversity work presented in this paper is the focus on one form of diversity. That is, whether naming, affirming, or removing barriers, there is a tendency to focus on one sociocultural marker of identity at a time. Even those with best intentions are neglecting to have a comprehensive lens of what it takes to diversify the field. "There is no such thing as a singleissue struggle because we do not live single-issue lives [48]." Black women and Women of Color physicists have, in particular, been calling for the use of intersectionality to consider simultaneous issues of gender, race, and other markers of difference that multiply-marginalized people face - even before the term intersectionality was coined [49, 50, 58-60]. For example, when considering disability, ableist assumptions about who is worthy of engaging physics has effects on representation at all levels of education and across race and ethnicity. While there are examples of physicists like Stephen Hawkings (a white man with cerebral palsy), seldom do we discuss or celebrate racially minoritized physicists with disabilities. For example, few know of Renee Horton who identifies as a female African American physicist with a disability [61] or Wanda Diaz Merced, a Puerto Rican astronomer with physical disabilities [62]. Understanding intersectionality can help those engaged in diversity work in PER to "identify praxis and processes that mitigate oppressive forces in education and the workplace [50]."

\section{B. Disrupting Tolerable differences in physics}

By complicating how diversity is framed and insisting that multiple aspects of oppression and/or marginalization be considered simultaneously, intersectionality as conceptualized by Black feminists can disrupt diversity work in physics that, in practice, limits the types of differences tolerable in the field. If, for example, the racially minoritized students who are named, affirmed, and for whom barriers are lifted also happen to perform to classed, gendered, and Eurocentric ex- pectations of what it means to do physics, then that diversity work is merely affirming dominant ways of being in the field. Similarly, if minoritized members of the PER community are expected to explain themselves and their scholarship to reviewers who privilege traditional approaches, then those scholars' marginalization will persist because the white, male, and westernized gaze will continue to be centered. As Patricia Hill Collins states, "oppressed groups are frequently placed in the situation of being listened to only if we frame our ideas in the language that is familiar to and comfortable for a dominant group. This requirement often changes the meaning of our ideas and works to elevate the ideas of dominant groups [5]."

\section{Collaboration}

Humans are not single issue people; so there should not be single issue discussions about the diversity of humans engaged in physics. There is an existing history of utilizing frameworks and interdisciplinary perspectives in PER [1] and doing so with a focus on equity may enhance efforts to address diversity. Increasing and enhancing collaborations with folx who address equity explicitly and use methodology and frameworks that are atypical in PER - such as scholars in multicultural education, special education, and urban education may be helpful. The PER community may benefit from collaborating more with experts in feminist studies, cultural studies, and critical theories.

\section{MOVING FORWARD}

As the field of physics moves forward there is a need to rethink how diversity work is conceptualized and implemented. Let us not replace the proverbial gatekeepers in our effort to transform the gate or open it wider. Let us tear down the gate. Let us not simply open the door to more kinds of differences. Let us tear down the door and chip away at the walls until they too are gone. Let our challenge be not that of deciding who should do physics, but rather let us meet the challenge of doing diversity by acknowledging, affirming, and removing barriers for all who have interest in doing physics.

\section{ACKNOWLEDGEMENTS}

The authors acknowledge and thank the PER community for doing the work of diversity in physics. The authors thank the collective group of People of Color in PER for doing the work in PER and physics. The authors thank those who reviewed this paper. Finally, the authors thank the 2020 PERC organizers, Alex V. Knaub, Lin Ding, Beth Cunningham, and Steven J. Maiers for organizing the panel discussion for which the first author was invited to submit a paper. 
[1] J. L. Docktor and J. P. Mestre, Phys. Rev. Phys. Educ. Res. 10, 2 (2014).

[2] L. C. Leathers, Ph.D. thesis, University of Iowa, 2010.

[3] P. J. Luginbuhl, E. H. McWhirter, and B. T. McWhirter, Journal of Latina/o Psychology 4, 1 (2016).

[4] L. Bush, L. Western Journal of Black Studies 23, 1 (1999).

[5] P. H. Collins, Black feminist thought: Knowledge, consciousness, and the politics of empowerment (Routledge, New York, 2000).

[6] K. Crenshaw, Stan, L. Rev. 43 (1990).

[7] The African American Policy forum website's page on Kimberlé Crenshaw, https://aapf.org/kimberle-crenshaw

[8] K. Crenshaw, The Washington Post, 24 (2015).

[9] E. Brewe and V. Sawtelle, Phys. Rev. Phys. Educ. Res. 12, 2 (2016).

[10] G. L. Cochran and G. D. White, Phys. Teach. 55, 6 (2017).

[11] G. L. Cochran and G. D. White, Phys. Teach. 58, 5 (2020).

[12] J. Blue, A. Traxler, and G. Cochran, Am. J. Phys. 87, 8 (2019).

[13] C. Prescod-Weinstein, Medium (February 27, 2017).

[14] I. J. Rodriguez, Medium (July 11, 2019).

[15] G. L. Cochran, The Scholarly Kitchen (June 22, 2018).

[16] A. Perry, Surge (March 29, 2019).

[17] Women in Physics, The Gazette - American Physical Society https://www.aps.org/programs/women/reports/gazette/index. $\mathrm{cfm}$

[18] Timothy J. Atherton, Ramon S. Barthelemy, Wouter Deconinck, Michael L. Falk, Savannah Garmon, Elena Long,Monica Plisch, Elizabeth H. Simmons, and Kyle Reeves. LGBT climate in physics: Building an inclusive community (American Physical Society, College Park, 2016).

[19] The AIP National Task Force to Elevate African Americans The time is now: Systemic changes to increase African Americans with bachelor's degrees in physics and astronomy (American Institute of Physics, College Park, 2020).

[20] Y. Le Espiritu, (Vol. 204, Temple University Press, Philadelphia, 1992).

[21] S. Hune, New Directions for Student Services 97 (2002).

[22] E. O'brien, The racial middle: Latinos and Asian Americans living beyond the racial divide (NYU Press, New York, 2008).

[23] AAPT Diversity, Equity, and Inclusion in Physics Page https://www.aapt.org/Resources/Physics-Diversity.cfm

[24] AAPT Her Stories Page https://www.aapt.org/resources/ herstories.cfm

[25] E. Cech and T. Waidzunas. STEM Inclusion Study Organization Report: AAPT (University of Michigan, Ann Arbor, 2017).

[26] E. Cech and T. Waidzunas. 2018. âSTEM Inclusion Study Organization Report: APS (University of Michigan, Ann Arbor, 2018).

[27] M. Boveda and A. B. Aronson, Remedial and Special Education, 40,4 (2019).

[28] A. Traxler and J. Blue Disability in Physics: Learning from Binary Mistakes. In Physics Education and Gender, (Springer, Cham, 2020).

[29] E. Scanlon, J. Schreffler, W. James, E. Vasquez, and J. J.Chini, J. J. Phys. Rev. PER 14, 2 (2018).

[30] W. James, K. Lamons, R. Spilka, C. Bustamante, E. Scanlon, and J. Chini, PERC 2019 Proceedings (2019).

[31] E. Scanlon and J. J. Chini, PERC 2018 Proceedings (2018).
[32] E. Scanlon, and J. J. Chini, PERC 2019 Proceedings, (2019).

[33] G. L. Cochran, T. Hodapp, and E.E. Brown PERC 2017 Proceedings, (2018).

[34] S. Nieto and P. Bod, Affirming diversity: The sociopolitical context of multicultural education (Pearson, Hoboken, 2018).

[35] Popsugar page - I'm Mexican American, but I won't Call Myself Chicana page https://www.popsugar.com/news/WhatDifference-Between-Mexican-American-Chicano-44728331

[36] N. Ackerman, T. Atherton, A. R. Avalani, C. A. Berven, T. Laskar, A. Neunzert, D. S. Parno, M. Ramsey-Musolf, LGBT+ inclusivity in physics and astronomy: A best practices guide, (arXiv.org, 2018)

[37] G. L. Cochran, A. Gupta, S. Hyater-Adams, A. V. Knaub, B. Zamarripa Roman, Emerging Reflections from the People of Color (POC) at PERC Discussion Space (arXiv.org, 2019).

[38] https://www.aip.org/diversity-initiatives/diversity-statement

[39] https://www.aapt.org/aboutaapt/organization/laboratories.cfm

[40] https://www.aps.org/programs/speakers/

[41] http://eblur.github.io/scotus/

[42] https://www.supremecourt.gov/oral_arguments/argument_tran script/2009list

[43] https://www.particlesforjustice.org/

[44] https://www.shutdownstem.com/about

[45] C. Prescod-Weinstein, Medium (November 6, 2017).

[46] C. Prescod-Weinstein, Medium (November 22, 2017).

[47] H. D. Lasswell, H. D. Politics: Who gets what, when, how. (Pickle Partners Publishing, 2018).

[48] A. Lorde, Learning from the 60s, in Sister Outsider: Essays and Speeches (Crossing, Berkeley, 2007).

[49] C. Prescod-Weinstein, Nature Astronomy, 1, 6 (2017).

[50] G. L. Cochran, M. Boveda, and C. Prescod-Weinstein Intersectionality in STEM Education Research. In Handbook of Research on STEM Education. Eds. C. Johnson, M. MohrSchroeder, T. Moore, and L. English (Routledge, New York, 2020).

[51] C. Miller and K. Stassun, Nature 510, 7504 (2014).

[52] C. W. Miller, B. M. Zwickl, J. R. Posselt, R. T. Silvestrini, and T. Hodapp, Science Advances 5, 1 (2019).

[53] J. R. Posselt, T. E. Hernandez, G. L. Cochran, and C. W. Miller, Journal of Women and Minorities in Science and Engineering 25, 4 (2019).

[54] AAPT Statement on Use of GRE for Admission to Graduate Physics Programs. Retrieved from https://www.aapt.org/aboutaapt/organization/diversity.cfm

[55] APS Bridge Program - Program Goals Page https://www.apsbridgeprogram.org/about/goals.cfm

[56] ETS Guidelines for the Use of Scores page https://www.ets.org/gre/institutions/admissions/using _scores/guidelines?WT.ac=40361_owt19_180820

[57] G. Potvin, D. Chari, and T. Hodapp, Physical Review - Physics Education Research 13, 2 (2017).

[58] G. L. Cochran (Ed) Fall 2019 Issue of the APS Gazette.

[59] S. M. Malcom, P. Q. Hall, and J. W. Brown, The double bind: The price of being a minority woman in science(American Association for the Advancement of Science, Washington DC, 1975)

[60] M. Ong, C. Wright, L. Espinosa,and G. Orfield, Harvard Educational Review 81, 2 (2011). 
[61] Throomers Dr. K. Renee Horton: World Class NASA Scientist page https://throomers.com/dr-k-renee-horton-world-classnasa-scientist/
[62] Wanda Díaz Merced https://royalsociety.org/topicspolicy/diversity-in-science/scientists-with-disabilities/wandadiaz-merced/ 